\title{
2. Max Halbes Theorie der »Prosalyrik« im Kontext der naturalistischen Neuordnung der Gattungshierarchie
}

Parallel zu Liliencrons praktischen Vorstößen in Richtung einer ,Prosaisierung der Lyrik ereignete sich in der zweiten Hälfte der achtziger Jahre bei den Vertretern des deutschen Naturalismus auch eine lebhafte theoretische Debatte über die Zukunft der Versdichtung in der Moderne. Die besondere Virulenz dieses Themas resultiert im wesentlichen daraus, daß damit beileibe nicht nur das Problem historischen Gattungswandels verhandelt wurde, sondern das Fortbestehen eines ganzen Ausdrucksmodus - und damit die Persistenz des Literatursystems in seiner bisherigen Form - auf dem Spiel stand. Zum ersten Mal in der Geschichte war der Status der Dichtung selbst unsicher geworden. ${ }^{1}$ Unmittelbarer Anlaß für diese grundsätzliche Infragestellung waren zwei zeitgenössische Entwicklungen, welche die Literatur nicht nur wesentlicher Funktionen der Weltdeutung beraubten, sondern auch das seit der Antike festgeschriebene poetologische Prinzip mimetischer Wirklichkeitsabbildung mit einem Mal obsolet erscheinen ließen: der Siegeszug der exakten Naturwissenschaften ${ }^{2}$ und die Erfindung neuer medialer Darstellungstechniken. ${ }^{3}$ Die

1 Kolkenbrock-Netz sieht denn auch die naturalistische Bewegung als „Versuch zur Rekonstituiening der Literatur als ,Kunst، unter ,kunstfeindlichen gesellschaftlichen Produktionsbedingungen«s Jutta Kolkenbrock-Netz: Fabrikation - Experiment - Schöpfung. Strategien ästhetischer Legitimation im Naturalismus. Heidelberg: Winter 1981 (= Reihe Siegen 28), S. 67.

2 Borchmeyer konstatiert zu Recht den "Totalitätsanspruch» naturwissenschaftlicher Theoriebildung im späten 19. Jahrhundert, der, indern mdie Induktion experimentell-positivistischer Herkunftu zur favorisierten Methode erklärt wird, faktisch einen "Wissenschaftsmonismus" begründe, welcher mallein die exakte Naturwissenschaft als Erkenntnisgrundlage anerkenntes, Dieter Borchmeyer: Der Naturalismus und seine Ausläufer. In: Viktor Żmegač (Hrsg.): Geschichte der deutschen Literatur vom 18. Jahrhundert bis zur Gegenwart. Bd. 2. Königstein i.Ts.: Athenäum 1980, S. 166 und 168. Auch Richter, Schönert und Titzmann gehen davon aus, daß das Erkenntnismodell 'Wissenschaft، in der zweiten Hälfte des 19. Jahrhunderts eine "Dominanz im Diskurssystem» erreicht, Karl Richter/Jörg Schönert/Michael Titzmann: Literatur - Wissen - Wissenschaft. Überlegungen zu einer komplexen Relation. In: K. R./J. S./M. T. (Hrsg.): Die Literatur und die Wissenschaften 1770-1930. Walter Müller Seidel zum 75. Geburtstag. Stuttgart: M \& P Verlag für Wissenschaft und Forschung 1997, S. 26.

3 Welche Rolle die modernen Bildaufzeichnungsmedien Daguerrotypie und Photographie in den ästhetischen Debatten nach 1850 spielten, hat vor allem Plumpe minutiös rekonstru- 
Dichtung mußte angesichts dieser Herausforderungen nun ihre weitere Existenzberechtigung überprüfen und sah sich dazu aufgerufen, grundsätzlich zu klären, welche Rolle sie in einer zunehmend durchrationalisierten Lebenswelt spielen konnte und sollte. $^{4}$

Um den veränderten ästhetischen Funktionsbedingungen zu entsprechen, schien es angeraten, die Literatur künftig am Leitbild der Naturwissenschaft auszurichten und deren Erkenntnisziele und methodische Prinzipien zu übernehmen. So forderte etwa Wilhelm Bölsche, die Poeten sollten ihre wveralteten Grundanschauungen [...] durch neue, der exacten Wissenschaft entsprechende» ersetzen, und sprach sich nachdrücklich für seine Anpassung« der Literatur san die neuen Resultate der Forschung “ ${ }^{5}$ aus. Dieser Paradigmenwechsel hatte weitreichende Konsequenzen, war es doch nicht damit getan, naturwissenschaftliche Verfahrensweisen künstlerisch zu adaptieren; vielmehr mußte die Literatur in toto auf die Axiome des szientifischen Weltbilds umgestellt werden. Die damit einhergehende Verabschiedung metaphysischer Prämissen ${ }^{6}$ machte letztlich eine Neubegründung der gesamten Ästhetik erforderlich, die nicht mehr länger transhistorisch verankert werden konnte. ${ }^{7}$ Conrad Alberti erklärte in diesem Sinne, daß, ebensowenig wie sewig geltende Wahrheiten

iert; vgl. Gerhard Plumpe: Der tote Blick. Zum Diskurs der Photographie in der Zeit des Realismus. München: Fink 1990. Siehe in diesem Zusammenhang aber auch Erwin Koppen: Literatur und Photographie. Über Geschichte und Thematik einer Medienentdeckung. Stuttgart: Metzler 1987.

4 Scheuer spricht von einer "Legitimationskrise" der Geistes- und Sozialwissenschaften; Helmut Scheuer: Naturalismus und Naturwissenschaft. In: Fin de siècle. Zu Naturwissenschaft und Literatur der Jahrhundertwende im deutsch-skandinavischen Kontext. Vorträge des Kolloquiums am 3. und 4. Mai 1984, hrsg. von Klaus Bohnen, Uffe Hansen und Friedrich Schmöe. Kopenhagen/München: Fink 1984 (= Text \& Kontext. Sonderreihe 20; Kopenhagener Kolloquien zur deutschen Literatur 11), S. 14.

5 Wilhelm Bölsche: Die naturwissenschaftlichen Grundlagen der Poesie. Prolegomena einer realistischen Ästhetik. Mit zeitgenössischen Rezensionen und einer Bibliographie der Schriften W. B.s neu hrsg. von Johannes J. Braakenburg. München: Deutscher Taschenbuch Verlag / Tübingen: Niemeyer $1976(=\mathrm{dtv}-$ Wissenschaftliche Reihe 4269; Deutsche Texte 40), S. 5.

6 So verkündete Bölsche 1887 programmatisch: "Wir haben gebrochen mit der Metaphysik.» Ebd., S. 48. Zur Metaphysikkritik im deutschen Naturalismus siehe Silvio Vietta: Neuzeitliche Rationalität und modeme literarische Sprachkritik. Descartes - Georg Büchner - Amo Holz - Karl Kraus. München: Fink 1981, S. 135-142.

7 Alberti etwa äußert das »tiefste Mißtrauen« gegenüber einer »alle Probleme metaphysisch lösenden Aesthetik» und erteilt deshalb den whochtönenden, spekulativen Begriffsspielereien der alten Aesthetiku eine klare Absage; Conrad Alberti [eigentlich: Sittenfeld]: Der Realismus als Weltanschauung. In: C. A.: Natur und Kunst. Beiträge zur Untersuchung ihres gegenseitigen Verhältnisses. Leipzig: Wilhelm Friedrich o.J. [1890], S. 23. Und Wilhelm Bölsche versieht seine 1890 im Kritischen Jabrbuch erschienene Besprechung von Eduard Hartmanns Philosophie des Schönen mit dem programmatischen Untertitel „Eine Grabrede auf die schematisirende Aesthetik der Gegenwart《. 
außerhalb der Mathematik ${ }^{8}$ existierten, auch keine weinheitlichen absoluten ästhetischen Begriffeu denkbar seien:

So wenig es ein Nirwana oder ein Wolkenkukuksheim oder einen Himmel giebt, so wenig giebt es ein absolutes Schönheitsideal, das als solches von Anbeginn der Welten bestände [...]. Die Schönheitsideale sind grundverschieden bei den Menschen verschiedener Zeiten, Gesellschaftsklassen, Ländem. ${ }^{9}$

Aus der Einsicht in die historisch-kulturelle Gebundenheit ästhetischer Ausdrucksmittel plädierte Alberti methodisch für einen konsequenten »Uebergang von der Spekulation zur philosophisch-geordneten Erfahrungswissenschaft» und damit für den Aufbau einer mneuen induktiven Aesthetik« unter der Leitformel der »Reduktion ${ }^{10}$.

Freilich gelang die angestrebte "Szientifizierung der Kunst ${ }^{11}$, durch welche die naturwissenschaftliche Methodik in handhabbare künstlerische Verfahrensweisen transformiert werden sollte, im Grunde nur beim Roman (Zola) und bei kürzeren Prosaformen (Holz, Schlaf), in gewisser Weise vielleicht auch noch beim Drama (Holz, Schlaf, Hauptmann). Als nahezu resistent gegenüber allen Versuchen, Wissenschaft und Literatur kurzzuschließen, erwies sich dagegen - wie kaum anders zu erwarten - die Lyrik, weil diese nach wie vor als ästhetisches Reservat metaphysischen Denkens fungierte. Das Diesseitigkeitspostulat und der Verzeitlichungsimperativ moderner Wissenschaft bedeuteten mithin einen Frontalangriff auf ihr Selbstverständnis. Während die übrigen Bereiche des Gattungsspektrums im wesentlichen nur konventionalisierte künstlerische Techniken durch Darstellungsverfahren ersetzen mußten, die sich an den analytischen Naturwissenschaften orientieren, schien die geforderte Verabschiedung transzendenter Grundlagen der Literatur für die Lyrik einer Selbstaufgabe gleichzukommen, bedeutete doch ein Verzicht auf metaphysische Letztbegründung der Dichtkunst vermeintlich auch den völligen Verlust spoetischer Aura.

Verschärft wurde die "kulturelle Legitimationskrise der Lyrik ${ }^{12}$ in den achtziger Jahren noch durch die nach wie vor bestehende Dominanz epigonaler Verseschmiede. Die naturalistischen Autoren sahen sich deshalb in einen ständigen rhetorischen Abwehrkampf gegen die mediokren Hervorbringungen der Gründerzeitlyriker gedrängt. Dementsprechend geißelte etwa Hermann Conradi das sepigonenhafte Scha-

8 Conrad Alberti: Ziele und Aufgaben der modernen Dichtung. In: C. A.: Natur und Kunst. Beiträge zur Untersuchung ihres gegenseitigen Verhältnisses, S. 216.

9

Conrad Alberti: Alte und neue Ästherik. In: ebd., S. 10 und 5f. Deshalb sei auch »das Schönheitsgefühl nichts apriorisches«; Conrad Alberti: Der Zweck in der Kunst. In: ebd., S. 114.

10 Conrad Alberti: Alte und neue Ästhetik. In: ebd., S. 4, 11 und 4.

11 Dieter Borchmeyer: Der Naturalismus und seine Ausläufer, S. 166.

12 Jutta Kolkenbrock-Netz: Fabrikation - Experiment - Schöpfung. Strategien ästhetischer Legitimation im Naturalismus, S. 110. 
blonenthum ${ }^{13}$ der poetischen Produktion seiner Zeit, das letztlich nur igleißende, aber in sich morsche und haltlose Fabrikarbeit ${ }^{14}$ entstehen lasse. Karl Henckell wandte sich vehement gegen die lyrischen "Phrasendrescher und Reimpolterer und konstatierte: "Wie auf allen übrigen Gebieten der Poesie ohne Ausnahme hat auch auf dem der Lyrik der Dilettantismus jeder Form das unrühmliche Scepter erobert. ${ }^{15}$ Die Ursache hierfür sah Julius Hart in einer bis in die Romantik zurückreichenden generellen "Loslösung der Form vom Inhalte، ${ }^{16}$; sie habe auf die Dauer zu einem sterilen „Formalismus« ${ }^{17}$ geführt und sei ganz wesentlich dafür verantwortlich, daß gegenwärtig »die dürrste Reimerei [...] für Poesie genommen « ${ }^{18}$ werde. Die verschiedenen geäußerten Einwände zusammenfassend zog Otto Julius Bierbaum schließlich 1891 Bilanz:

Keine Gattung der deutschen Dichtung ist so ins Kunsthandwerk ausgeartet, wie die Lyrik, keine auch ist in der allgemeinen Achtung so gesunken. [...] Die Quantität ist ins unermeßliche [sic] gestiegen, die Qualität abgrundtief gesunken. Wie das Klavierspielen ist die Lyrik unter die Dilettanten gerathen und zu einer fast gemeingefährlichen Klimperkunst geworden. Sie, die eine Kunst der Herzenskündigung sein soll, also die Kunst kühnster Eigenoffenbarung, ist conventionell, also eigenartslos geworden [...]. Sie ist die ebenbürtige Schwester derjenigen Sorte von Genremalerei, welche man mit dem anmutigen Einsilber Kitsch sehr schön bezeichnet: leer und gefällig. ${ }^{19}$

Uneinigkeit bestand allerdings darin, wie dem hohen Automatisierungsgrad der zeitgenössischen Gedichtproduktion angesichts des zunehmenden Bedeutungsverlustes der Lyrik angemessen zu begegnen sei. Je nachdem, ob die Dignitätseinbuße der Versdichtung als temporäres und damit überwindbares Auslaugungsphänomen oder als definitiver Endpunkt eines langen historischen Entwicklungsprozesses interpretiert wurde, bildeten sich zwei gegensätzliche Fraktionen heraus. Während die eine ihr Augenmerk auf eine Erneuerung lyrischer Ausdrucksmittel richtete, suchte die andere die Notwendigkeit einer Umschichtung des Gattungsgefüges in Richtung auf

13 Hermann Conradi: Unser Credo. In: Modeme Dichter-Charaktere, hrsg. von Wilhelm Arent. Mit Einleitungen von Hermann Conradi und Karl Henckell. Berlin: Selbstverlag des Herausgebers (In Commission der Kamlah'schen Buchhandlung) 1885, S. III. Ebd., S. II.

15 Karl Henckell: Die neue Lyrik. In: ebd., S. VI und V.

16 Julius Hart: Homo sum! Ein neues Gedichtbuch. Nebst einer Einleitung: Die Lyrik der Zukunft. Großenhain/Leipzig. Verlag von Baumert \& Ronge 1890, S. XI.

17 Ebd., S. X. Rückblickend wertet auch Busse wdie Schönfärberei und den Formalismus» als verhängnisvollste Erscheinungen »der älteren Prosak; Karl [sic] Busse: Die moderne Bewegung in der deutschen Literatur. In: Tägliche Rundschau, Unterhaltungsbeilage, Nr. 215, 12.9.1896, S. 858 .

19 Otto Julius Bierbaum: Deutsche Lyrik von heute. Vortrag, gehalten am ersten öffentlichen Abend der Gesellschaft für modemes Leben. Mit einem Anhang: Über die von der Gesellschaft für modernes Leben geplanten Sonderausstellungen von Werken der bildenden Kunst. München: Poeßl 1891 (= Münchener Flugschriften 2), S. 5. 
die narrative Erzählprosa theoretisch zu begründen. Die Gruppe der lyrischen Reformer sah sich besonders durch das Erscheinen der Gedichte Detlev von Liliencrons in ihrer Einschätzung bestätigt, schienen seine Texte doch erstmals Wege aufzuzeigen, wie sich die epigonale Rückständigkeit der deutschen Poesie überwinden ließ. Sein Beispiel regte denn auch zahlreiche Nachahmer dazu an, selbst mit Versdichtungen an die Öffentlichkeit zu treten, und so kam es ab Mitte der achtziger bis zum Beginn der neunziger Jahre zu einem ungeahnten Aufschwung der Lyrik bei den Vertretern der naturalistischen Bewegung. ${ }^{20}$ Vor allem die Freiheiten, welche sich Liliencron in Wortwahl, Syntax, Metrik und Reimtechnik herausnahm - offensichtliche Regelverstöße gegen hergebrachte Normen bewußt einkalkulierend -, wirkten als Anreiz, die herkömmlichen Grenzen künstlerischen Ausdrucks zu erweitern. Infolgedessen orientierte sich nicht nur die meue Lyrik " ${ }^{21}$, die von Schriftstellern im Umkreis des Naturalismus vorgelegt wurde, in mancherlei Hinsicht deutlich an dessen literarischen Verfahrensweisen, auch die sie begleitenden programmatischen Stellungnahmen richteten sich mit ihren zentralen Forderungen am Vorbild seiner Texte aus.

Die »Parole [...], daß die ,Wahrheit< wichtiger sei als die $\$$ Schönheit ${ }^{22}$, bot dabei eine willkommene Legitimation für die Überschreitung bisheriger ästhetischer Lizenzen. Starre, aus dem Traditionsfundus normativer Poetik stammende Regeln ebenso wie konventionalisierte lyrische Darstellungsmuster konnten durch den Hinweis auf die Authentizität subjektiven Ausdrucksbestrebens mindestens partiell auBer Kraft gesetzt werden. So propagierte beispielsweise Hermann Conradi 1885 eine "schrankenlose, unbedingte Ausbildung « der »künstlerischen Individualität ${ }^{23}$ als probates Gegenmittel gegen jede Art von ästhetischer Epigonalität. Ganz ähnlich empfahl Carl Bleibtreu im selben Jahr: "In allererster Linie muß die Subjektivität entfesselt werden, um die Erstarrung in konventioneller Schablone zu brechen. ${ }^{24}$ Im Grunde griffen die Naturalisten mit solchen Forderungen ziemlich unverhüllt auf Argumentationsmuster der Genieästhetik, besonders des Sturm und Drang, zurück. ${ }^{25}$ Wie

20 Bleibtreu konnte schon im Jahre 1886 konstatieren, daß meine neue Blütheperiode der Lyrik angebrochen sei; Carl Bleibtreu: Revolution der Literatur. Mit erläutemden Anmerkungen und einem Nachwort neu hrsg. von Johannes J. Braakenburg. Tübingen: Niemeyer 1973 (= Deutsche Texte 23), S. 67. Vgl. hierzu generell die monographische Darstellung von Jürgen Schutte: Lyrik des deutschen Naturalismus (1885-1893). So die Überschrift von Karl Henckells Einleitungsaufsatz zur ersten naturalistischen Lyriksammlung Modeme Dichber-Charaktere (1885). Roy C. Cowen: Der Naturalismus. In: Hans Joachim Piechotta/Ralph-Rainer Wuthenow/ Sabine Rothemann (Hrsg.): Die literarische Moderne in Europa. Bd. 1: Erscheinungsformen literarischer Prosa um die Jahrhundertwende. Opladen: Westdeutscher Verlag 1994, S. 69. Hermann Conradi: Unser Credo, S. III. 
deutlich dieser Rekurs auf die Zielsetzungen der ersten deutschen Literaturrevolte ${ }^{26}$ war, zeigt sich besonders an der Gattungspräferenz: Die Hochschätzung der Lyrik in der Frühphase des deutschen Naturalismus - sie verdrängte bei einigen Autoren gar das Drama von der Spitze der Hierarchie literarischer Ausdrucksformen ${ }^{27}$ - verweist eben nicht nur deutlich auf den besonderen Stellenwert, welcher der Artikulation von Subjektivität ganz generell eingeräumt wurde, sondern indiziert auch eine grundsätzliche Übereinstimmung mit "Zentralanliegen der Sturm und Drangepocher $^{28}$.

Um dem individuellen Gestaltungswillen des jeweiligen Autors größtmöglichen Spielraum zu geben, sprachen sich die naturalistischen Theoretiker vor allem für die Nutzung formal wenig reglementierter Genres aus. Im Bereich der Lyrik hatte dies einen weitgehenden Verzicht auf herkömmliche Poetizitätsmerkmale wie Reim, Strophe und Metrum zur Folge. Viele Schriftsteller griffen die von den Harts ausgesprochene Empfehlung, "Poeme [...] in freierer, reimloser Form ${ }^{29}$ zu verfassen, auf,

Rückwärtsgewandte der naturalistischen Modernerc Helmut Koopmann: Die Klassizität der "Modeme«. Bemerkungen zur naturalistischen Literaturtheorie in Deutschland. In: Beiträge zur Theorie der Künste im 19. Jahrhundert. Hrsg. von Helmut Koopmann und J. Adolf Schmoll gen. Eisenwerth. Bd. 2. Frankfurt a.M.: Klostermann 1972 (= Studien zur Philosophie und Literatur des neunzehnten Jahrhunderts 12/2), S. 148. Kolkenbrock-Netz spricht dagegen präziser von einer "Emeuenung der Geniekonzeption des 18. Jahrhunderts«; Jutta Kolkenbrock-Netz: Fabrikation - Experiment - Schöpfung. Strategien ästhetischer Legitimation im Naturalismus, S. 118. Beispielhaft vorgebildet ist das Geniepathos der frühen Naturalisten in Heinrich und Julius Harts Aufsatz Friedrich Spielhagen und der deutsche Roman der Gegenwart, vgl. H. H./J. H.: Kritische Waffengänge. 6. Heft. Leipzig: Otto Wigand 1884, S. $73 f$.

Siehe hierzu Walter Schmitz: Literaturrevolten: Zur Typologie von Generationengruppen in der deutschen Literaturgeschichte. In: Kongreß Junge Wissenschaft und Kultur: Die Lebensalter in einer neuen Kultur? Zum Verhältnis von Jugend, Erwerbsleben und Aiter. Hrsg. von Rudolf Walter Leonhardt. Köln: Bachem 1984 (= Veröffentlichungen der Hanns-Martin-Schleyer-Stiftung 13), S, 144-165.

27 So erklärt Henckell sie zur mursprünglichsten, elementarsten und reinsten aller Dichtungsarten«; Karl Henckell: Die neue Lyrik, S. V.

28 Günter Mahal: Wirklich eine Revolution der Lyrik? Überlegungen zur literaturgeschichtlichen Einordnung der Anthologie Modeme Dichter-Charaktere, S. 20. Vortrag Deutsche Lyrik (1891) hebt Bierbaum von den »Veränderungen [...], welche [...] die lyrische Form» in der jüngsten Zeit merfahren hatı, denn auch besonders die Befreiung von Reim und Metrum hervor: "Ich weise in dieser Hinsicht nur auf das Eine hin, auf die ausgedehnte Verwendung der freien Rhythmik, welche, kurz gesagt, das Bestreben hat, als einziges Gesetz der Form den Inhalt anzuerkennen, so zwar, daß der Fluß der Verse sich lediglich nach dem Wesen der ausgedrückten Gefühle richtet. Auch hierin drückt sich die Abwendung vom rein äußerlichen [sic] und der Trieb nach anschaulicher Charakteristik aus.« Otto Julius Bierbaum: Deutsche Lyrik von heute, S. 10. Was von den Harts und Bierbaum so lobend herausgestrichen wird, erfährt freilich von Schriftstellem außerhalb des naturalistischen Gruppenzusammenhangs eine durchaus skeptische Bewertung. So mokiert 
und so erlebte die freirhythmische Dichtung seit Mitte der achtziger Jahre einen lebhaften Aufschwung. ${ }^{30}$ Dabei folgten die naturalistischen Lyriker freilich nur zu einem Teil der Traditionslinie von Klopstock, Goethe, Novalis und Hölderlin, die dadurch geprägt ist, die Preisgabe metrisch-strophischer Regulierung durch einen besonders restriktiven Umgang mit Lexik und Syntax zu kompensieren. ${ }^{31}$ Obwohl vereinzelt immer wieder auf die Register xerhabenen Sprechens zurückgegriffen wurde, richteten doch die meisten Autoren ihre Bemühungen darauf, unprätentiösere Wege der freirhythmischen Textgestaltung zu finden. Offenbar hatten sie erkannt, daß eine wirkliche sBefreiung، lyrischen Ausdrucks, um glaubhaft zu wirken, außer einem Abbau formaler Normierungen eben auch eine Lockerung sprachlicher Konventionen erfordert.

Da neben den freien Rhythmen in der deutschen Poetik bekanntlich nur noch eine einzige Versart existiert, die sich außerhalb festgelegter metrischer Regulierung bewegt, kann es kaum erstaunen, daß es im Zuge der naturalistischen Erneuerung der Lyrik auch zu einer Wiederbelebung des Knittelverses kam. Der sogenannte freie Knittelvers als paarig gereimter Vers mit variabler Silbenzahl bildete das "Haupt-Metrum epischer und dramatischer Dichtung “" ${ }^{32}$ des 15. und 16. Jahrhunderts, geriet dann nach Martin Opitz' Reform der Prosodie an den Rand des lyrischen Gattungssystems und überdauerte die Zeit bis zum Ende des 19. Jahrhunderts als nurmehr selten genutzte Sonderform dichterischen Ausdrucks. ${ }^{33}$ Gerade dieser

sich beispielsweise Karl Kraus in einer Rezension von Otto Emsts Neuen Gedichten über "gewisse $>$ Lyriker, die die Moderne gepachtet zu haben meinen, wenn sie den scheußlichsten Freirhythmenquark produzierenж; Die Gesellschaft 9 (1893), S. 509.

Leider hat diese signifikante Konjunktur, die im übrigen als weiterer Beleg für die Affinität der Naturalisten zur Sturm-und-Drang-Bewegung gelten kann, in den einschlägigen Darstellungen zur Geschichte der freien Rhythmen fast keine Berücksichtigung gefunden. Allenfalls Nietzsche und Holz werden in diesem Zusammenhang genannt, vgl. etwa Louis Benoist-Harnappier: Die freien Rhythmen in der deutschen Lyrik. Ihre Rechtfertigung und Entwicklung. Halle: Niemeyer 1905; August Closs: Die freien Rhythmen in der deutschen Lyrik. Versuch einer übersichtlichen Zusammenfassung ihrer entwicklungsgeschichtlichen Eigengesetzlichkeit. Bern: Francke 1947; L. [eif] L.[udwig] Albertsen: Die freien Rhythmen. Rationale Bemerkungen im allgemeinen und zu Klopstock; Bert Nagel: Der freie Vers in der modernen Dichtung. Göppingen: Kümmerle 1989 (= Göppinger Arbeiten zur Gemanistik 512). Eine Geschichte der freien Rhythmen in der literarischen Modeme wäre also erst noch zu schreiben.

31 Frey bemerkt hierzu: „In der deutschen Überlieferung sind die freien Rhythmen von Anfang an eine hymnische Form gewesen, geeignet, Erfahrungen zu machen, welche die Gemessenheit strenger Regelmäßigkeit durchbrechen und in Begeisterung münden.» HansJost Frey: Verszerfall, S. 32.

32 Christian Wagenknecht: Deutsche Metrik. Eine historische Einführung. München: C.H. Beck 1981 (= Beck'sche Elementarbücher), S. 40; vgl. hierzu auch Hans-Jürgen Schlütter: Der Rhythmus im strengen Knittelvers des 16. Jahrhunderts. In: Euphorion 60 (1960), S. 48-90.

33 Campe charakterisiert den Knittelvers folgendermaßen: meine Benennung der vor Opizens 
Nimbus des Unabgenutzten in Verbindung mit dem - nach allgemeiner Einschätzung - "(scheinbar) unkünstlerischen Charakter “ ${ }^{34}$ der Versart ließ sie für Autoren im Umkreis des Naturalismus wieder interessant werden. ${ }^{35} \mathrm{Im}$ Vergleich zu den freien Rhythmen bot der Knittelvers zudem den Vorteil, daß mit seiner Verwendung traditionell die niederer Stilebene konnotiert war. Er eignete sich daher in besonderem Maße als alternatives Ausdrucksmuster für eine Lyrik, die mit den herkömmlichen sprachlich-formalen Konventionen brechen wollte.

Nicht alle Naturalisten teilten jedoch den Glauben ihrer Kollegen an die Sinnhaftigkeit einer Neubelebung lyrischer Dichtkunst. Vielmehr sahen einige die Bedeutung der Versdichtung angesichts der Prädominanz der Naturwissenschaften und einer ständig komplexer werdenden sozialen Realität, die zunehmend vom Prinzip der Rationalität durchdrungen wird, zwangsläufig schwinden. Vor allem Carl Bleibtreu vertrat die Auffassung, daß sdie Enge der lyrischen Form sie untauglich macht, den ungeheuren Zeitfragen zu dienen ${ }^{36}$. Zugleich zeigte er sich überzeugt davon, daß sich sdie Lyrik [...] als Dichterberuf [...] überlebt ${ }^{37}$ habe. Im Grunde bediente er sich damit einer Argumentationsfigur, welche die ästhetische Debatte seit Schiller prägt, hatte dieser doch in seiner Abhandlung Über die ästhetische Erriebung des Menscben in einer Reihe von Briefen (1795) erstmals einen unmittelbaren Zusammenhang

Zeit gewöhnlichen kurzzeiligen und meist platten holperigen Verse, die nur der Reim zu Versen machte; dann, überhaupt, schlechte Verse, wo mehr auf das Reimen als auf die Fügung gesehen wird, und wo auch selbst die Reime schlecht und oft falsch sindk; Wörterbuch der Deutschen Sprache. Veranstaltet und hrsg. von Joachim Heinrich Campe. Bd. 2. Braunschweig: In der Schulbuchhandlung 1808, S. 991. Vgl. auch Otto Flohr: Geschichte des Knittelverses vom 17. Jahrhundert bis zur Jugend Goethes. Berlin: Vogt 1893 (= Berliner Beiträge zur germanischen und romanischen Philologie. Germanische Abteilung 1). Chistian Wagenknecht: Deutsche Metrik. Eine historische Einführung, S. 46.

In einem undatierten Brief an Dehmel vom Februar 1893 hebt Liliencron besonders die Variabilität "dieses Versmaßes« hervor: "Grade der Knittelvers ist so sehr modulationsfáhig.» Detlev von Liliencron: Ausgewählte Briefe, Bd. 1, S. 282. Neben dem Knittelvers gibt es zu dieser Zeit eine ganze Fülle von Experimenten, metrisch ungeregelte Verse in freier Weise mit Reimen zu kombinieren.

36 Carl Bleibtreu: Revolution der Literatur, S. 67. Dabei vertrat Bleibtreu in vielerlei Hinsicht eine recht traditionalistische Auffassung von Poesiec. Im Grunde war er eher bereit, auf den Ausdrucksmodus Lyrik ganz zu verzichten als ihn einem Wandlungsprozeß auszusetzen, der in Richtung einer ’Prosaisierung führen würde. So äußerte er in einer Rezension über Amo Holz' frühe freirhythmische Lyrik: wdiese naturalistische Prosa in Metren und Reimen werden wir nimmermehr als Poesie anerkennen'; zitiert nach Helmut Scheuer: Arno Holz im literarischen Leben des ausgehenden 19. Jahrhunderts (1883-1896). Eine biographische Studie. München: Winkler 1971 (= Winkler Studien), S. 53.

37 Carl Bleibtreu: Revolution der Literatur, S. 70. Ëine ähnliche Auffassung wie Bleibtreu vertritt Berg, wenn er konstatiert. "[...] in unserer Zeit [...] muss der Dichter der Sprache des gewöhnlichen Lebens lauschen, weil [...] er den Feind nicht anders als mit seinen eigenen Waffen schlagen kann - und diese Sprache ist dem Verse unzugänglich!» Leo Berg. Der Naturalismus. Zur Psychologie der modemen Kunst. München: Poeßl 1892, S. 116. 
zwischen den allgemein bestehenden lebensweltlichen Existenzbedingungen und den Möglichkeiten künstlerischer Artikulation hergestellt. Schillers grundsätzliche Diagnose jedenfalls wird letztlich auch noch von Bleibtreu geteilt:

Jetzt aber herrscht das Bedürfnis und beugt die gesunkene Menschheit unter sein tyrannisches Joch. Der Nutzen ist das große Idol der Zeit, dem alle Kräfte fronen und alle Talente huldigen sollen. Auf dieser groben Waage hat das geistige Verdienst der Kunst kein Gewicht, und, aller Aufmunterung beraubt, verschwindet sie von dem lärmenden Markt des Jahrhunderts. Selbst der philosophische Untersuchungsgeist entreißt der Einbildungskraft eine Provinz nach der andern, und die Grenzen der Kunst verengen sich, je mehr die Wissenschaft ihre Schranken erweitert. ${ }^{38}$

Im Anschluß an Schiller hatte Hegel dann erklärt, daß die Kunst als an die Sinnlichkeit gebundenes Darstellungs- und Erkenntnismedium in der Moderne von der rein begrifflich operierenden Philosophie endgültig abgelöst und damit zu einem geschichtsphilosophischen Durchgangsstadium herabgestuft werde. ${ }^{39}$ Was die Literatur betrifft, werde die >Poesier zudem immer stärker von der sich ausbreitenden Prosa an den Rand gedrängt, was ihre Stellung prekär werden lasse. ${ }^{40}$ Hegel und Schiller stehen denn auch als - ungenannte - Referenzgrößen im Hintergrund von Bleibtreus Argumentation, die sich allerdings nicht auf die Gesamtheit ästhetischer Gestaltungsformen bezieht, sondern lediglich auf eine bestimmte Dichtungsart. Aus der Gewißheit heraus, daß der Vers als Ausdrucksmittel historisch obsolet geworden sei, riet er seinen Kollegen unmißverständlich, sie mögen »Prosa scbreiben, wenn sie "etwas zu sagen babenw ${ }^{41}$. Auch wenn Bleibtreu der Lyrik ihre Existenzberechtigung

38 Friedrich Schiller: Sämtliche Werke. Auf Grund der Originaldrucke hrsg. von Gerhard Fricke und Herbert G. Göpfert. 9., durchgesehene Auflage. Bd. 5. München/Wien: Hanser 1993, S. 572.

Vgl. hierzu das berühmte Diktum über die Kunst: Sie "[...] ist und bleibt [...] nach der Seite ihrer höchsten Bestimmung für uns ein Vergangenes. Damit hat sie für uns auch die echte Wahrheit und Lebendigkeit verlorenc; Georg Wilhelm Friedrich Hegel: Werke, Bd. 13, S. 25.

40 Hegel bemerkt in diesem Zusammenhang. Tun sich die " $[. .$.$] poetischen Fordenungen nun$ in einer Zeit hervor, in welcher die bloße Richtigkeit der prosaischen Vorstellung schon zur gwohnten Norm geworden ist, so hat die Poesie [...] eine schwierigere Stellung. [...] Außerdem treten noch anderweitige Schwierigkeiten ein [...]. Wenn nämlich der prosaische Verstand schon an die Stelle der ursprünglich dichterischen Vorstellung getreten ist, so erhält die Wiedererweckung des Poetischen [...] leicht etwas Gesuchtes», wodurch die Literatur mallzu schnell ins Raffinieren und Haschen nach Wirkungen kommt«; ebd., Bd. 15, S. 281 283.

Carl Bleibtreu: Revolution der Literatur, S. 72. Schon Friedrich Schlegel hatte in seiner Schrift Über das Studium der griechischen Poesit (1795/97) erklärt: "Prosa ist die eigentliche Natur der Modemen. Friedrich Schlegel: Kritische Schriften und Fragmente. Studienausgabe in sechs Bänden, hrsg. von Emst Behler und Hans Eichner. Bd. 1. Paderborn/München/ Wien/Zürich: Schöningh 1988, S. 86. 1888 gab Bleibtreu gar seiner Hoffnung auf einen "Shakespeare des Romans« Ausdruck; Carl Bleibtreu: Der Kampf ums Dasein der Literatur. Leipzig: Wilhelm Friedrich 1888, S. 72. 
nicht schlechthin absprach, sondern ausdrücklich konzedierte: „Nebenber wird sie ewig ihre Berechtigung behalten, natürlich vorausgesetzt, dass sie wirklich echte vollquellende Melodie des Herzens, nicht aber eine Sprachübung für Dilettanten sei «" war sie damit doch als entwicklungsgeschichtliches Relikt abgestempelt.

Lange Zeit schienen die beiden konträren Positionen des Naturalismus zur Lyrik nicht miteinander vermittelbar. Die Vertreter der einen Richtung konnten zur Verteidigung ihrer Überzeugung auf die Innovationsleistungen neuerer Gedichtproduktionen verweisen, die Wortführer der anderen beriefen sich im Gegenzug auf die prinzipielle Geltungskraft ihrer geschichtsphilosophischen Diagnose, deren Richtigkeit durch kurzlebige ästhetische Konjunkturen nicht beeinträchtigt werde. Einen auch theoretisch anspruchsvollen Versuch, die Frage nach der Lebensberechtigung der Lyrik zu beantworten, ohne in die dichotomischen Argumentationsmuster der jüngsten Auseinandersetzungen zurückzufallen, unternahm erst Max Halbe. In expliziter Auseinandersetzung mit Bleibtreus Thesen ging er in seinem programmatischen Aufsatz Lyrik? $(1889 / 90)^{43}$ daran, Zukunftsperspektiven und Entwicklungs-

42 Carl Bleibtreu: Revolution der Literatur, S. 70. Deshalb läßt sich aus seiner provokant formulierten Standortbestimmung auch nicht einfach die Schlußfolgerung ziehen, daß mdie deutschen Naturalisten zunächst den Roman und die Erzählprosa [...] als die den theoretischen Implikationen adäquate Form begriffen « hätten, wird eine solch dichotomische Sicht der Dinge doch durch die umfangreiche Produktion lyrischer Texte widerlegt; Roy C. Cowen: Der Naturalismus, S. 91. Ob man freilich umgekehrt davon sprechen kann, wdaß die naturalistische Lyrik in ihrem wesentlichen Teil den distinkteren Konzeptionen des reigentlichen، Naturalismus zeitlich vorausgeht's wie Schütte dies tut, wäre erst noch genauer zu prüfen; Jürgen Schurte: Lyrik des deutschen Naturalismus (1885-1893), S. 14.

43

So heißt es zu Beginn des Textes explizit: „Die Lyrik ist das Schmerzenskind des modemen Naturalismus. Man ist soweit gegangen, überhaupt ihre Daseinsberechtigung anzuzweifeln. Walter Hettche: Max Halbes Berliner Anfänge. Mit unveröffentlichten Texten aus dem Nachlaß. In: Textgenese und Interpretation. Vorträge und Aufsätze des Salzburger Symposions 1997. Hrsg. von Adolf Haslinger, Herwig Gottwald und Hildemar Holl. Stuttgart: Akademischer Verlag Hans-Dieter Heinz 2000 (= Stuttgarter Arbeiten zur Germanistik 389; Salzburger Beiträge 41), S. 59. Die Tatsache, daß Bleibtreu zum Zeitpunkt der Entstehung des Aufsatzes Mitherausgeber der Gesellschaft - des bis dahin konkurrenzlosen publizistischen Zentralorgans der naturalistischen Bewegung - war, läßt es verständlich werden, warum Halbe die Gründung der Zeitschrift Freie Bühne für modernes Leben zum Anlaß nahm, um dort seine Gegenposition zu publizieren. Jedenfalls schickte er seinen Lyrik? Essay gleich im Januar 1890, unmittelbar nachdem er zur Mitarbeit an dem neuen Periodikum aufgefordert worden war, an den Redakteur Amo Holz. Dieser sandte den Text jedoch mit der Begründung wieder zurück, er sei mzu lang" und win seiner Form etwas zu radicalic; ebd., S. 59. Offensichtlich blieb der Essay ungedruckt. Dennoch muß auch ohne öffentliche Verbreitung davon ausgegangen werden, daß die von Halbe formulierten Thesen in den Kreisen der Naturalisten eine gewisse Bekanntheit erlangten. Zumindest wurde dort über ähnliche Problemstellungen intensiv diskutiert, so daß Halbes Position jenseits aller positivistischen Einflußforschung in jedem Fall als eine Art imaginäre Bezugsgröße herangezogen werden kann. (Vgl. auch Kapitel III/6.) 
möglichkeiten der Versdichtung angesichts der zunehmend kunstfeindlichen Bedingungen der zeitgenössischen Gegenwart auszuloten. Vor die eigentliche Prognose stellte er dabei eine Wesensbestimmung des lLyrischen und unterschied in diesem Zusammenhang zwischen einem überzeitlichen, anthropologisch im Ausdrucksbedürfnis des Menschen »begründeten Kerm der Lyrik« und ihren wveränderlichen $u$. dem Wechsel der Zeit unterworfenen «Erscheinungsformem ${ }^{44}$. Um ihn tatsächlich als Invariable begreifen zu können, umreißt Halbe den "Kern « nur höchst abstrakt: »Lyrik [...], auf das Bleibende $u$. Unzerstörbare an ihr untersucht, ist weiter nichts als die dichterische Wiedergabe subjectiver Gefühle und Stimmungen, gleichgiltig, in welche Form dieselben gegossen werden. ${ }^{45}$ Diese allgemein gehaltene Definition leistet zweierlei: Zum einen dient sie dazu, Halbes Ansatz theoretisch abzustützen, wird damit doch direkt an die neuere Gattungspoetik seit Goethe, den Schlegels und Hegel angeschlossen, die übereinstimmend Lyrik zum literarischen Ausdrucksmedium von Subjektivität erklärt hat. ${ }^{46}$ Zum anderen aber gibt der bewußt reduktive Charakter dieser Bestimmung Halbe die Gelegenheit, alle anderen Merkmale, die lyrischem Sprechen gewöhnlich zugeschrieben werden, zu bloßen Akzidentien herabzustufen:

Weder der Rhythmus noch der Reim sind also integrirende Bestandtheile der Lyrik oder bilden ,Grundgesetzer derselben [...] wie unsere Aesthetikbücher glauben machen wollen. Rhythmus $u$. Reim sind die Erscheinungsformen, in denen die heutige Menschheit die Lyrik zu erblicken gewohnt ist, die sie darum mit dem Kem verwechselt. Sie stammen aus der Kindheit der Menschheit, $u$. es ist alle Wahrscheinlichkeit, daß sie mit dieser Kindheit verschwinden werden. 47

Gemeinhin als basal angesehene Formelemente lyrischer Texte wie Metrum und Reim werden so zu beliebig verfügbaren Kunstmitteln erklärt, deren Präsenz oder Absenz letztlich keinen Einfluß auf den Gattungsstatus hat. Wenn aber das gesamte Arsenal bisheriger lyrischer Verfahrensweisen geschichtlich wandelbar ist, dann bestimmt sich auch das Verhältnis von Vers- und Prosatexten neu. Anders gesagt: Sobald Lyrik nicht mehr als Dichtung in sgebundener Reder begriffen wird, erscheint

44 Ebd., S. 62. Er knüpft damit natürlich in gewisser Weise an Goethes in den Noten und $A b$ handlungen $x^{u}$ besserem Verständris des West-östlichen Divans (1819) getroffene Unterscheidung zwischen »Naturformen der Dichtung« und "Dichtarten» an. Halbes Gedankengang kehrt gut fünfzig Jahre später in abgewandelter Form in Staigers »Fundamentalpoetik« (S. 12, 232) wieder, wenn »die Zufälligkeit der äußeren Erscheinung eines Gedichts» (S. 229) vom "Wesen des Lyrischen« (S. 7$)$ abgehoben wird, das nur durch munbeirrbare Ideation" (S. 207) aufzufinden sei; die Seitenangaben beziehen sich auf die Ausgabe: Emil Staiger: Grundbegriffe der Poetik [1946]. Zweite erweiterte Auflage. Zürich: Atlantis Verlag 1951. Walter Hettche: Max Halbes Berliner Anfänge, S. 62.

46 Vgl. in diesern Zusammenhang vor allem Dorothea Ruprecht: Untersuchungen zum Lyrikverständnis in Kunsttheorie, Literarhistorie und Jiteraturkritik zwischen 1830 und 1860.

Walter Hettche: Max Halbes Berliner Anfänge, S. 62. 
lyrischer Ausdruck auch in Prosa möglich. ${ }^{48}$ Halbe geht sogar noch weiter, deutet er die sProsaisierung der Lyrik doch als notwendige Folge eines übergreifenden literarischen Entwicklungsprozesses. So wie sich in "Eipik u. Dramatik« schon seit geraumer Zeit der Übergang von sgebundener zu sungebundener Rede vollzogen habe, sei eine analoge Entwicklung auch bei der dritten dichterischen Fundamentalgattung unausweichlich. Allerdings stecke sdieser Prozeß bei der Lyrik noch in seinen Anfängen«:

Es widerstrebt uns, lange Gespräche oder Erzäblungen in rhythmischer Form zu hören, aber einem Jeden von uns, und sei er der Vorgeschrittenste, setzen sich gewisse erhabene Augenblicksstimmungen noch in Rhythmik um. [...]

So kommt es, daß selbst die Wenigen, deren Wirklichkeitssinn entwickelt genug ist, um die Prosaform theoretisch als das lyrische Zukunftsideal aufzustellen, sich practisch dem Klangzauber freier Rythmen [sic], ja selbst dem eines Reimgedichtes nicht zu entziehen vermögen. $^{49}$

Es sei freilich nur eine Frage der Zeit, bis diese durch ästhetische Normenbildung und soziale Habitualisierung verankerten Prägungen ihre Kraft verlieren würden. Die gegenwärtig noch bestehenden Vorbehalte gegenüber der Prosa würden deshalb auch nichts an der allgemeinen Gültigkeit von Halbes Prognose ändern, die da lautet: »Die Prosaform ist die Zukunft der Lyrik, wie jeder dichterischen Wirkung. " ${ }^{50}$

48 Ähnliche Überlegungen wie Halbe stellte etwa zur gleichen Zeit auch Julius Hart an. In seinem Aufsatz Der Kampf um die Form in der zitgenörsischen Dichsung (1890) erklärt er, daß das "Wesen der dichterischen Sprache [...] nicht in Reim und Rhythmus eingeschlossen wirft im Anschluß daran die Frage auf: "Sollte es daher nicht eine dichterische Sprache geben, die ihren eigentlichen und wahren Zweck auch ohne Zuhülfenahme dieser mächtigen Mittel erreicht? Literarische Manifeste des Naturalismus 1880-1892. Hrsg. von Erich Ruprecht. Stuttgart: Metzler 1962, S. 182. Allerdings limitiert er den Galtungsrahmen der Prosa sogleich wieder, indem er einschränkend hinzufügt, "daß die ungebundene Rede ausschließlich in Zeiten des Veberganges zum Ausdruck der Dichtkunst wirdk; ebd., S. 187. Dieses Argumentationsmuster wird in der Folgezeit eine beliebte Formel, die dazu dient, das Prosagedicht zu einem temporären Durchgangsstadium der Literaturentwicklung zu erklären. Walter Hettche: Max Halbes Berliner Anfänge, S. 63.

50 Ebd. Halbe führt hier augenscheinlich einen Gedanken fort, der bei Scherer vorformuliert ist. So heißt es in dessen postum erschienener Poetik. (1888) explizit: „Man könnte sich [...] noch mancherlei Gattungen construiren, mancherlei denken was in Wirklichkeit bis jetzt nicht eingetreten [...]. Namentlich ist es denkbar, daß es eine vollständige Lyrik in Prosa gäbe. Alle lyrischen Gattungen könnten auch in Prosa versucht werden ohne den Zwang von Metrum und Reim."Wilhelm Scherer: Poetik. Mit einer Einleitung und Materialien zur Rezeptionsanalyse. Hrsg. von Gunter Reiß. Tübingen: Deutscher Taschenbuch Verlag / Niemeyer 1977 (= Deutsche Texte 44), S. 25. Als historische Beispiele derart "prosaischer Lyriki führt Scherer die biblischen Psalmen und die Prosafassung von Novalis' Hymnen an die Nacht an; ebd., S. 24. Zumindest punktuell scheint es also Berührungspunkte zwischen den poetologischen Verortungsversuchen einzelner moderner Autoren und der akademisch betriebenen Ästhetik gegeben zu haben. Daß Halbe, der sich seit Oktober 1885 in Berlin aufhielt, in diesem und im darauffolgenden Jahr eifrig Scherers Lehrveranstaltungen besuchte, 
Halbe rekurriert mit seiner Argumentation geschickt auf die von Hamann, Herder und Hegel vertretene These, wonach die Lyrik einen geschichtlich sehr frühen Stand der Menschheitsentwicklung widerspiegle. ${ }^{51}$ Anders als diese zieht er daraus jedoch nicht den Schluß, die Versdichtung könne auf Grund ihrer Ursprünglichkeit besondere Dignität beanspruchen, sondern fordert statt dessen, daß das »Kindheits«-Stadium literarischen Ausdrucks endlich überwunden werden müsse. Die Prosa wird von ihm denn auch nicht als Verfallserscheinung im Vergleich zu einer angenommenen ursprünglichen Vollkommenheit gesehen, ${ }^{52}$ sondern als komplexes Produkt, das sich in einem langwierigen Ausdifferenzierungsprozeß herausgebildet habe. Er antizipiert damit in gewisser Weise Lotmans Prosaverständnis, das ja auf der Annahme basiert, daß sdie Prosa, ungeachtet ihrer scheinbaren Einfachheit und ihrer Nähe zum gewöhnlichen Sprachgebrauch, ästhetisch komplizierter als die Poesiex ${ }^{53}$ sei.

geht aus seinen Erinnerungen hervor; vgl. Max Halbe: Scholle und Schicksal. Die Geschichte meiner Jugend. Neue, durchgesehene und überarbeitete Ausgabe. Salzburg. Verlag "Das Bergland-Buch" 1940, S. $316 \mathrm{f}$.

51 Hamann bezeichnet zu Beginn seiner Aesthetica in nuce (1762) die "Poesien als »die Muttersprache des menschlichen Geschlechts«; Johann Georg Hamann: Sämtliche Werke. Historisch-kritische Ausgabe, hrsg. von Josef Nadler. Bd. 2: Schriften über Philosophie, Philologie, Kritik 1758-1763. Wien: Herder 1950, S. 197. Herder seinerseits zeigt sich in der $A b$ bandlung über den Unsprung der Sprache (1772) überzeugt, daß die »Poesie älter [...] als Prosacs sei, und folgert daraus, "dic erste Sprache des menscblichen Geschlecbts sei Gesang genesemuc Johann Gottfried Herder: Werke in zehn Bänden. Hrsg. von Martin Bollacher [...]. Bd. 1: Frühe Schriften 1764-1772. Hrsg. von Ulrich Gaier. Frankfurt a.M.: Deutscher Klassiker Verlag 1985 (= Bibliothek deutscher Klassiker 1), S. 740. Ähnlich steht für Hegel fest: "Die Poesie ist älter als das kunstreich ausgebildete prosaische Sprechen. Sie ist das ursprüngliche Vorstellen des Wahren«; Georg Wilhelm Friedrich Hegel: Werke, Bd. 15, S. 240. Eine derartige Ansicht war freilich unter den Theoretikem des Naturalismus nicht unangefochten. So sprach etwa Alberti in seinem Aufsatz Kunst und Daninismus die gegenteilige Vermutung aus: „Höchst wahrscheinlich ist die Poesie aus der Prosa entstanden durch eine Art natürlicher Auslese der bedeutungsvollsten Wörter" Conrad Alberti: Alte und neue Ästhetik, S. 79. Alberti folgt damit der Argumentationslinie, die Charles Batteux in seiner Abhandlung Les Beaux: Ants réduit à un méme principe (1747) begnïndet hatte.

52 Diese Auffassung prägt fast durchweg das Prosaverständnis des 18. Jahrhunderts; vgl. hierzu etwa Hans-Otto Rößer. Bürgerliche Vergesellschaftung und kulturelle Reform. Studien zur Theorie der Prosa bei Johann Gottfried Herder und Christian Garve. Frankfurt a.M./ Bern/New York: Lang 1986 (= Gießener Arbeiten zur neueren deutschen Literatur und Literaturwissenschaft 9).

53 Jurij M. Lotman: Vorlesungen zu einer strukturalen Poetik, S. 59. Vorgebildet ist diese Ansicht freilich schon im 19. Jahrhundert. So heißt es etwa bei Berg: wIst die Prosa [...] prosaischer als der Vers, weil sie komplizierter, zusammengesetzter ist? Ist, was dem prähistorischen Menschen ein ungeheueres Complexum von Vorstellungen war, auch den modemen Menschen ein gleich Mannigfaltiges? Der Rhythmus des Verses ist einfacher, die Prosa mit ihrem durchgebildeten Satzgefühl setzt ein feineres Unterscheidungsvermögen, das Umfassen grösserer Vorstellungsgebiete und Gedankenreihen voraus. Aber umfasst dergleichen 
Im Endeffekt bestätigt Halbe zwar die Prämisse von Bleibtreus Prognose, weist aber dessen Schlußfolgerungen zurück. Fraglos würden die durch die aktuellen Entwicklungen in Wissenschaft und Technik bedingten Umwälzungen der Lebensverhältnisse auch auf die Literatur durchschlagen und letztlich - wie schon von Hegel vorhergesagt und später von der jungdeutschen Ästhetik bestätigt ${ }^{54}-$ zu einer Dominanz der Prosa im Gattungsspektrum führen. Dies bedeute aber keineswegs, daß die Lyrik sich als Ausdrucksmedium überlebt habe. Halbes Resümee lautet deshalb: „Die Erscheinungsformen der Lyrik werden sich wandeln u. zusammendrängen. Aber die Opposition, die sich gegen die Daseinsberechtigung der Lyrik selbst wendet, schießt über ihr Ziel hinaus. Sie [...] verwechselt den Kern des Dinges mit seinen Erscheinungsformen. "55 Auch wenn die Lyrik - bezogen auf die Gesamtheit literarischer Gestaltungsweisen - künftig an Bedeutung verlieren werde, würden sich ihr doch zugleich im Rahmen der Prosa ungeahnte Wirkungsmöglichkeiten erschließen. Als gelungenes Beispiel für diese neuartige »Prosalyrik« nennt Halbe Turgenevs Gedichte in Prosa, die den "Reimdurchfällen zahlreicher Reimkünstler" ${ }^{56}$ weit überlegen seien. Damit waren nun auch Liliencrons Versuche einer Annäherung der Lyrik an die Prosa, wie sie vor allem in der Integration von Prosatexten in den Kontext einer Gedichtsammlung und durch die Verbindung von Vers- und Prosapassagen innerhalb eines Textes zum Ausdruck kommen, nachträglich theoretisch legitimiert. ${ }^{57}$

Halbes Versuch, den Geltungsbereich der Lyrik neuzudefinieren, ist in mehrfacher Hinsicht bemerkenswert. Im Gegensatz fast zur gesamten deutschsprachigen Poetik seiner Zeit löst er sich vom althergebrachten Dogma, daß das ,Wesen lyrischer Dichtung von Vertextungskonventionen wie Reim und Metrum bestimmt

der Verstand eines modemen Menschen nicht? Nimmt er nicht mehr und nicht Grösseres auf einmal in sein Bewusstsein auf als der prähistorische Mensch im Vergleich zum Tiere? Ist das Reich seiner Vorstellungen nicht mächtiger, mannigfaltiger geordnet, beziehungsreicher? « Leo Berg: Der Naturalismus. Zur Psychologie der modemen Kunst, S. 117.

$54 \mathrm{Vgl}$. in diesem Zusammenhang etwa Mundts These von der fortschreitenden „Emancipation der Prosau; Theodor Mundr: Die Kunst der deutschen Prosa, S. 49. Siehe auch Wolfram Malte Fues: Poesie der Prosa, Prosa als Poesie. Eine Studie zur Geschichte der Gesellschaftlichkeit bürgerlicher Literatur von der deutschen Klassik bis zum Ausgang des 19. Jahrhunderts.

Walter Hettche: Max Halbes Berliner Anfänge, S. 64.

56 Ebd. Daß Halbe das neue Gattungsmodell nicht nur theoretisch umrissen, sondem mindestens in einem Fall auch praktisch erprobt hat, zeigt der Text Das bist $D_{4}$. Ein Gedicht in Prosa, der 1891 in der Münchner Zeitschrift Modeme Blätter erschien; er ist wiederabgedruckt in: ebd,, S. 64f. In Halbes Erinnerungen heißt es später rückblickend über diese Zeit: »Ich experimentierte damals viel herum, schrieb Skizzen, Studien, versuchte mich in rhythmischer Prosar; Max Halbe: Scholle und Schicksal. Die Geschichte meiner Jugend, S. 372.

57 Daß Halbe die Adjutantenritte kannte, geht aus seinen Lebenserinnerungen hervor. Dort weist er darauf hin, daß die darin enthaltenen Texte meine vollständig neu entdeckte Weltı für ihn gewesen seien; ebd., S. 301. 
würde. ${ }^{58}$ Ihm gelingt auf diese Weise eine konsequente Historisierung und Entmythisierung lyrischer Verfahrensweisen. Und obwohl auch er grundsätzlich von einer anthropologischen Verankerung der literarischen Fundamentalgattungen ausgeht, schreibt er diese doch weit weniger normativ fest als die übrigen zeitgenössischen Theoretiker. ${ }^{59}$ Damit erweist sich Halbes Ansatz in der Komplexität seiner Argumentation dem Bleibtreus als überlegen. Geht letzterer lediglich vom aktuellen Bestand literarischer Formen aus und sieht selbst dieses Arsenal in Zukunft weiter schrumpfen, vermag Halbe auch das Aufkommen neuer, bislang noch nicht dagewesener Gestaltungsmuster zu imaginieren. Zudem denkt er die Grenzen zwischen den einzelnen Ausdrucksbereichen als prinzipiell durchlässig. Er integriert daher wie selbstverständlich ein Phänomen wie das Prosagedicht in den Gesamtzusammenhang der Lyrik. Dies ist angesichts der im Deutschland der Jahrhundertwende noch allgemein verbreiteten Auffassung, daß die Lyrik eine der drei "Naturformen der Dichtung đarstelle, deren ästhetischer Zuständigkeitsbereich ein für alle Mal festgelegt sei, ein bemerkenswerter Schritt.

Halbes Gedankenführung hat indes einen unübersehbaren Schwachpunkt: Er liegt in der Identifizierung lyrischen Ausdrucks mit der wdichterischen Wiedergabe subjectiver Gefühle u Stimmungen ${ }^{60}$. Wenn man nämlich die Artikulation von Subjektivität zum einzigen Ausweis lyrischen Sprechens erklärt, dann droht die gesamte Lyrik seltsam ortlos zu werden, wird man doch weder der Erzählprosa noch dem Drama das Element des Subjektiven wirklich absprechen wollen. Auch die Postulierung eines gattungsspezifischen Anteils von Subjektivität führt hier nicht weiter, bleibt doch immer die Frage bestehen, wie dieser Prozentsatz gegebenenfalls zu bestimmen ist - ganz abgesehen von der Schwierigkeit, woran sich denn eigentlich zuverlässig erkennen läßt, daß in einem Text ssubjective Gefühle u Stimmungen« wie-

58 Ähnlich radikal verfährt dann erst wieder Amo Holz, wenn er erklärt: »Als formal Letztes in jeder Lyrik, das überhaupt uneliminierbar ist, bleibt für alle Ewigkeit der Rhythmus. Reim, Strophe, Parallelismus, Alliteration und Assonanz - man könnte noch beliebig fortfahren - waren nur akzessorisch und mußten daher mit der Zeit als systemer notgedrungen abwirtschaften. ( (W X, S. 510) Vgl. Kapitel III/6.

59

So erwägt selbst ein so unkonventioneller Denker wie Wilhelm Bölsche emsthaft, ob nicht weine unvertilgbare, ewig wieder hervorbrechende Neigung für die Verssprache» sin uns stecktı, die den Menschen waus rhythmischen Gründen« dazu nötige, möglicherweise sogar entgegen seinen Willen und wider bessere Erkenntnis am dichterischen Ausdruck in gebundener Sprache festzuhalten.« Wilhelm Bölsche: „Hinaus über den Naturalismus! Ein Wort an die Siebenmeilenstiefler in der Kunst. In: Freie Bühne für modernes Leben 1 (1890), S. 1049. Und auch Julius Hart zeigt sich in seinem Aufsatz Zur litterarischen Bezegung der Gegenwart (1886) überzeugt: „Die ewigen Gesetze, das ewig gülrige Wesen der Poesie besteht heute ebenso gut, wie ehemals, daran kann niemals gerürtelt werden.« Zitiert nach: Jutta Kolkenbrock-Netz: Fabrikation - Experiment - Schöpfung. Strategien ästhetischer Legitimation im Naturalismus, S. 70.

Walter Hettche: Max Halbes Berliner Anfänge, S. 62. 
dergegeben werden. Der Umstand, daß Halbe diese Probleme trotz der ihnen innewohnenden Brisanz nonchalant übergeht, verweist freilich darauf, daß sein Fokus nicht auf eine theoretische Begründung der Lyrik gerichtet ist, sondern auf den Nachweis ihrer geschichtsphilosophischen Berechtigung. Um dieses Ziel zu erreichen, nimmt er in seinen Ausführungen ohne zu zögern auch partielle logische Inkonsistenzen in Kauf.

Damit entpuppt sich Halbes Aufsatz im Kern als Verteidigungsschrift - gerichtet gegen jene fundamentale Infragestellung der Lyrik, die Carl Bleibtreu in propagandistischer Absicht unternommen hatte. Um dessen These vom unabwendbaren Niedergang der Poesie zu widerlegen, entwirft der Verfasser ein Argumentationsmodell, welches das künftige Weiterbestehen lyrischer Ausdrucksformen legitimiert. Nicht der Roman sei dasjenige Genre, auf welches die weitere Entwicklung der Literatur unaufhaltsam zulaufe; ${ }^{61}$ vielmehr erfasse der Prozeß der $>$ Prosaisierung، alle Bereiche des Gattungsspektrums. Dies bedeute aber keineswegs das Ende der Lyrik, sondern führe lediglich zu einem - wenn auch einschneidenden - poetischen Formenwandel. Lyrik ist von nun an nicht mehr länger als Dichtung in igebundenerc Sprache definiert, sondern vermag auch in Prosagestalt aufzutreten. Halbes Theorie der »Prosalyrik» öffnet so das Gattungsspektrum für neuartige Formen literarischen Ausdrucks, bindet ein Genre wie das Prosagedicht implizit zugleich aber wieder an die Gestaltungstradition der spoetischen Prosac zurück. ${ }^{62}$ Offen bleibt bei seinem gewagten Manöver in jedem Fall, wie Poetizität jenseits räßerlichı-formaler Signale wirkungsvoll codiert werden soll - eine ebenso heikle wie drängende Frage, die denn auch in den gesamten neunziger Jahren des vorletzten Jahrhunderts ihre Virulenz behält. Antworten darauf zu finden überläßt Halbe kurzerhand seinen Kollegen. Auch wenn dies seine Leistung als Literaturtheoretiker schmälem mag, kommt ihm doch zumindest insofern eine historisch bedeutsame Rolle zu, als er das ungewohnte Phänomen des Prosagedichts nachhaltig in die Entwicklungsgeschichte der Lyrik integriert hat. ${ }^{63}$ Von nun an existieren im deutschsprachigen Raum zwei unterschiedli-

61 So dekretiert Bleibtreu kurzerhand: "Die höchste Gattung des Realismus ist der sociale Romanw; Carl Bleibtreu: Revolution der Literatur, S. 36. Ähnlich versteht auch Alberti den Roman als "die Dichtform des darwinistischen Zeitalters«; Conrad Alberti: Zur Technik des modernen Romans. In: C. A.: Alte und neue Ästhetik, S. 242.

Derartige "Kompromifjormen des ästhetischen Diskurses« sind charakteristisch für die deutsche Literaturtheorie im 19. Jahrhundert insgesamt, die Innovationen durchweg an vorhandene Traditionsbestände anzukoppeln sucht, und kennzeichnen - anders als KolkenbrockNetz meint - durchaus nicht nur die Poetik des Naturalismus; Jutta Kolkenbrock-Netz: Fabrikation - Experiment - Schöpfung. Strategien ästhetischer Legitimation im Naturalismus, S. 112.

63 Wie sehr die Konzeption einer "Prosalyrik» den damaligen ästhetischen Diskurs polarisiert hat, können zwei konträre Stellungnahmen aus dem Umkreis des Naturalismus verdeutlichen. So verteidigt etwa Leo Berg die von Halbe propagierte Lockerung der lyrischen Formkonventionen nachdrücklich: "Es ist wahrhaftig keine Bequemlichkeit der modernen 
che, zuweilen in Konflikt zueinander tretende Deutungsmuster der Gattung. Während die einen das Prosagedicht als spezifische Form der Kurzprosa sehen, das mit der Versdichtung allenfalls den geringen Umfang und die Tendenz zur komprimierten Aussage gemein hat, verstehen es die anderen als spezifisch moderne Ausprägungsform der Lyrik, die zwar vom äußeren Erscheinungsbild an die Prosa erinnert, dies aber durch ihren poetischen، Gehalt wieder gänzlich kompensiert. ${ }^{64} \mathrm{Daß}$ beide Klassifizierungen im Grunde das Resultat hochgradig ideologisch besetzter Wertzuschreibungen sind, liegt auf der Hand. Nur deshalb aber konnte die Auseinandersetzung um die Textform in den folgenden Jahren ein Ausmaß erreichen, durch welche das Prosagedicht zur wohl wichtigsten und einflußreichsten Genreinnovation des ausgehenden 19. Jahrhunderts avancierte. ${ }^{65}$

Auch in Halbes Versuch, das Weiterbestehen der Lyrik geschichtsphilosophisch zu rechtfertigen, spiegelt sich die Ambivalenz der frühmodernen Poetologie in Deutschland wider. Einerseits propagiert er mit dem Prosagedicht eine historisch neuartige Gattung, andererseits nimmt er ihr von vornherein ihren potentiell subversiven Charakter, indem er sie pauschal der Lyrik zurechnet. Die postulierte Innovation wird also durch die Subsumtion unter das triadische Gattungsmodell sogleich wieder entschärft. Dies belegt einmal mehr die "Janusköpfigkeit« ${ }^{66}$ der naturalisti-

Dichter, wenn sie sich vom L'erse emanzipieren! Mit welch' tieferem Emst wird heute von den Prosaikern gearbeitet! Das feinere Ohr, die grössere Kunst ist bei ihnen. Die hervorragendsten modernen Dichtungen sind jedenfalls nicht in Versen geschrieben. . Leo Berg. Der Naturalismus. Zur Psychologie der modernen Kunst, S. 115f. Er vertritt sogar die Ansicht, "dass häufig gerade von seiten der Formverletzer die höhere Wirkung ausgeht», weil "gerade hier die grössere Zucht herrschtw; ebd., S. 115. Julius Hart dagegen lehnt alle Bestrebungen, den Vers für obsolet zu erklären, kategorisch ab: „Der sicherste Beweis für die volle Zerrüttung unserer Versform und unseres Formgefühls liegt in der Thatsache, daß man heute weithin in literarischen Kreisen den Vers überhaupt für eine Form der Vergangenheit ansieht, jede Versdichtung von vornherein mit Mißwollen als eine sunmoderner Schöpfung ansieht und nur noch die Prosa als für die Dichtung zulässig ansieht.« Julius Hart: Homo sum! Ein neues Gedichtbuch, S. XII.

Allerdings gab es zu dem Zeitpunkt, zu dem Halbe seinen Aufsatz verfaßte, auch in Frankreich schon zahlreiche Spielarten siyrisierter Kurzprosa; vgl. hierzu Suzanne Bemard: Le poème en prose de Baudelaire jusqu'à nos jours, S. 361-406.

Auch wenn Nolting-Hauff terminologisch in unzulässiger Weise simplifiziert, erkennt sie den gemeinten Sachverhalt doch genau: „Das Prosagedicht war um 1890 das modeme lyrische Genus par excellence.. Ilse Nolting-Hauff: Prousts $A$ la recherche du temps perdu und die Tradition des Prosagedichts. In: Poetica 1 (1967), S. 73. Ortlieb spricht in diesem Zusammenhang zu Recht, obgleich reichlich lapidar, von der wModewelle der deutschen Prosagedichte in den 90-er Jahren des 19. Jahrhunderts«; Comelia Ortlieb: Poetische Prosa. Beiträge zur modemen Poetik von Charles Baudelaire bis Georg Trakl, S. 17.

Jutta Kolkenbrock-Netz: Fabrikation - Experiment - Schöpfung. Strategien ästhetischer Legitimation im Naturalismus, S. 69. Schon Koopmann hatte zuvor vom »]anusgesichtigen« der mnaturalistischen Epoche« gesprochen; Helmut Koopmann: Die Klassizität der 
schen Bewegung, die vor allem daraus resultiert, daß ihre Vertreter meinen Bruch in der Traditionslinie der Literatur" propagieren, während sie ideologisch und verfahrenstechnisch erkennbar an wergangene Literaturepochen ${ }^{67}$ anknüpfen. Indem die rhetorische Erneuerungsemphase, die aus vielen Texten spricht, mit einer stillschweigenden Revitalisierung ästhetischer Normenkomplexe aus der Vergangenheit einhergeht, werden die Auswirkungen des lebensweltlichen Modernisierungsschubs auf die künstlerischen Verfahrensweisen systemverträglich abgefedert.

»Moderner. Bemerkungen zur naturalistischen Literaturtheorie in Deutschland, S. 140 sowie - ähnlich - auch S. 147.

67 Jutta Kolkenbrock-Netz: Fabrikation - Experiment - Schöpfung. Strategien ästhetischer Legitimation im Naturalismus, S. 69. Zugleich ist eine winnere Affinität zwischen den Tendenzen Jüngstdeutschlandss und der gegenmodemen Literaturbewegung um und nach 1900k zu konstatieren; Emst Ribbat: Propheten der Unmittelbarkeit. Bemerkungen zu Heinrich und Julius Hart. In: Wissenschaft als Dialog. Studien zur Literatur und Kunst seit der Jahrhundertwende. [Wolfdietrich Rasch zum 65. Geburtstag.] Hrsg. von Renate von Heydebrand und Klaus Günther Just. Stuttgart: Metzler 1969, S. 61. 